Dhaka Univ. J. Biol. Sci. 23(2): 187-195, 2014 (July)

\title{
ADAPTATION OF ITEMS OF BAYLEY SCALES OF INFANT DEVELOPMENT-II (BSID-II) SUITABLE FOR BANGLADESHI INFANTS
}

\author{
Monowara Parveen ${ }^{1}$, Syed Tanveer Rahman*, Shaheen Islam², Sultana \\ SARWATARA Zaman ${ }^{3}$, Jena HAMAdANI ${ }^{4}$ AND Naila Zaman KHAN ${ }^{5}$ \\ Department of Psychology, University of Dhaka, Dhaka-1000, Bangladesh
}

Key words: Scale adaptation, Infant development, Bayley scales, Bangladeshi infants

\begin{abstract}
The purpose of this study was to culturally adapt the items of Bayley Scales of Infant Development-II (BSID-II) for administration upon Bangladeshi infants. The adaptation procedure was conducted by following four steps: (i) field survey on normative sample, (ii) national expertise opinion, (iii) international expertise consultancy and (iv) picture sensitivity review. The culture based problems of the original BSID-II were identified from the initial field survey. Major problems identified by the examiners were unfamiliarity of the children with the items (pictures, words and materials). These assessments were evaluated by the national and international consultants. The uncommon items were changed to culturally recognizable ones while Bangla words were replaced to make the test culture-fair. At last, the adapted test was given to 22 healthy children to assess picture sensitivity of the test and found suitable where the infants attentively responded to the items.
\end{abstract}

\section{Introduction}

There are many factors that affect children's development ${ }^{(1)}$ e.g. poverty, nutritional deficiencies, lack of stimulating environment, maternal depression, institutionalization, etc.(2). It is therefore important to use appropriate tools for psychological assessment of children so that, early intervention and treatment can be introduced in time according to their developmental needs to prevent impairment and disabilities.

Assessment of children's development requires specific psychometric tests and is dependent on culture and practices of each country. In Bangladesh, there are not so many psychometric tests that are standardized to assess Bangladeshi children's development. So, the first crucial step is to adapt the psychometric tests based on cultural variation in the country. However, it requires sophistication to develop any culture-free test. Most researchers suggest to design culture-fair tests, drawn from experiences found in many cultures $^{(3)}$. When a psychometric test is developed in one country and administered into

*Author for correspondence: <tan@univdhaka.edu>. ${ }^{1}$ Dhaka Shishu Hospital, Dhaka. ${ }^{2}$ Department of Educational and Counseling Psychology, University of Dhaka, Dhaka-1000, Bangladesh. ${ }^{3}$ Institute of Education and Research, University of Dhaka, Dhaka-1000, Bangladesh. ${ }^{4}$ ICDDR,B, Mohakhali, Dhaka. ${ }^{5}$ Child Health Institute, Dhaka Shishu Hospital, Dhaka, Bangladesh. 
another with a different culture, it usually creates unfamiliarity, difficulty and culture biasness. The same experience occurs when western psychometric tests are administered on Bangladeshi infants and children from eastern cultural background.

Many of the prominent and widely used psychometric tests are developed in English language making difficult for non-English speaking children to understand and comprehend the test items and materials. These tests such as the revised version of Bayley Scales of Infant Development (BSID-II) ${ }^{(4)}$ used for assessing Bengali population is needed to be translated into Bengali language for it to be comprehended properly. Children's learning and development depend on their environment, so in new situations children face difficulties to adapt as the previous learning hinders the new learning. Therefore, keeping in mind the particular cultural characteristics and social norms and removing all cultural variations a psychometric test may be adapted from one culture to another.

In Bangladesh, many risk factors are responsible for delayed development of a child which is frequently identified in the health clinics or hospitals. Therefore, it badly needs to have culture sensitive psychometric tests for Bangladeshi infants and children.

The objective of the present study is cultural adaptation of the items and materials of the BSID-II for Bangladeshi infants and children.

\section{Materials and Methods}

The adaptation of Bayley Scales of Infant Development-II (BSID-II) involved four steps: (i) field survey on a normative sample, (ii) national experts' opinion, (iii) international experts' consultancy and (iv) picture sensitivity review following crosssectional survey design.

The original version of BSID-II was administered on a sample of incidentally selected 55 Bangladeshi children (male $=35$, female $=15$ ), with normal development aged between 1 and 42 months and 5 disabled children (male $=3$, female $=2$ ).

A total of 30 experts purposively selected at the national level were addressed for their valuable opinion for adaptation of BSID-II. Among them ten were child psychologists, ten were pediatricians, and the rest ten were developmental therapist and preschool teachers.

Two visiting foreign child psychologists (following convenience sampling technique) were approached for giving opinion on the adaptation process.

To assess the picture sensitivity of the modified BSID-II a sample of 22 children (from three to four years of age) were selected following incidental sampling technique. The distribution of sample is shown in Table 1.

The Bayley Scales of Infant Development (BSID-II) was published in 1993. The former version of BSID was published in 1969. BSID-II is an individually administered 
examination that assesses the current developmental functioning of infants and children. It consists of three scales: i.e. (i) mental scale (cognitive, personal-social and language development), (ii) motor scale (gross and fine motor development) and (iii) behavior rating scale - BRS assesses the child's behavior during the test situation. The three scales are considered complimentary to each other as well as each makes a unique combination for the evaluation of the children.

Table 1. Participants of the present study.

\begin{tabular}{|c|c|c|}
\hline Participants & No. & Description \\
\hline \multirow{3}{*}{$\begin{array}{l}\text { Infants and children- } \\
\text { ( } 1-42 \text { months age range) }\end{array}$} & & Disabled \\
\hline & \multirow[b]{2}{*}{55} & 3 boys \\
\hline & & 15 girls \\
\hline Developmental psychologists & 3 & $\begin{array}{l}\text { Working at the Child Development Center (CDC) of } \\
\text { Dhaka Shishu Hospital for field studies had } \\
\text { administration skill on BSID-II }\end{array}$ \\
\hline International consultants visited & 2 & \multirow{2}{*}{$\begin{array}{l}\text { Reviewer and consultant of BSID-II from the USA and } \\
\text { Developmental Paediatrics, Institute of Child Health, } \\
\text { UK }\end{array}$} \\
\hline CDC of Dhaka Shishu Hospital & & \\
\hline \multirow[t]{2}{*}{ Expert's opinion (national) } & \multirow[t]{2}{*}{30} & $\begin{array}{l}10 \text { paediatrics, } \\
10 \text { child psychologists }\end{array}$ \\
\hline & & $\begin{array}{l}10 \text { developmental therapists at CDC Dhaka Shishu } \\
\text { Hospital and teachers of a Kindergarten school at } \\
\text { Dhaka }\end{array}$ \\
\hline Picture sensitivity test & 22 & $\begin{array}{l}3 \text { to } 4 \text { years old children of Play-group (Kindergarten } \\
\text { students) and siblings of OPD and IPD patients at } \\
\text { Dhaka Shishu Hospital }\end{array}$ \\
\hline
\end{tabular}

In the field survey, the original BSID-II was administered by three trained developmental psychologists at Child Development Centre of Dhaka Shishu Hospital. Most of the participants were selected from the hospital's waiting room and were the siblings of the patients that came to the children hospital and pediatric clinic from urban and rural sectors. Some of the participants were children of the staffs of Dhaka Shishu Hospital and local area day-care centres. The test results were evaluated so that the items (pictures and materials) that were not found suitable for Bangladeshi infants were modified by an artist based on the suggestions given by the other group of 30 experts with child related experience namely ten child psychologists, ten pediatricians, ten developmental therapists and preschool teachers. In addition, two international experts their valuable opinion on the responses given by the children. The modified version was administered by three developmental psychologists on 22 children ( 3 to 4 years old) to assess the picture sensitivity of BSID-II. 


\section{Results and Discussion}

From the field survey, three particular parts of BSID-II were found to be difficult for the Bangladeshi children to comprehend - pictures, materials and words. The changes were kept to minimum possible requirement and the concept and difficulty level were maintained.

A total of 30 pictures were failed to be recognized by Bangladeshi sample and were therefore modified. These were dog, leaf, fish, house, leaves, etc. (Figs 1 and 2).

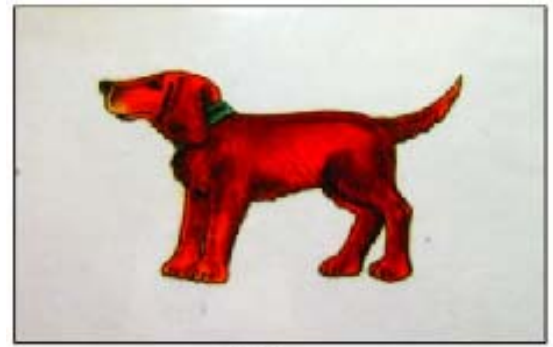

Dog

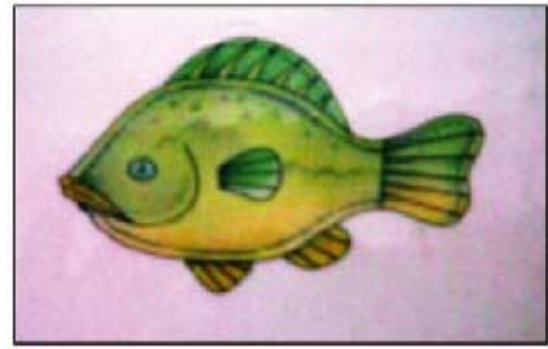

Fish (Pomfret)

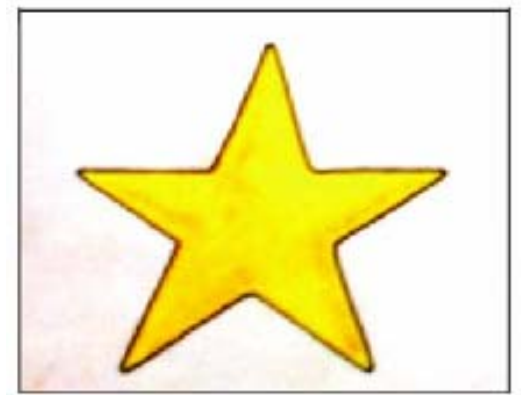

Star (unfamiliar picture for Bangladeshi children)

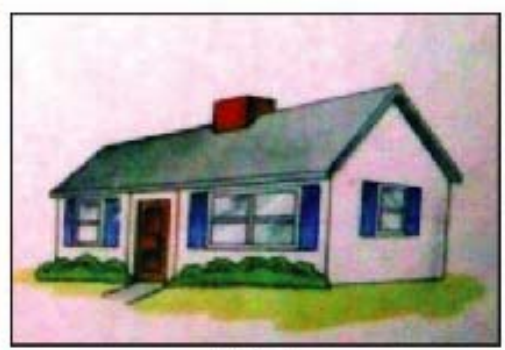

Home

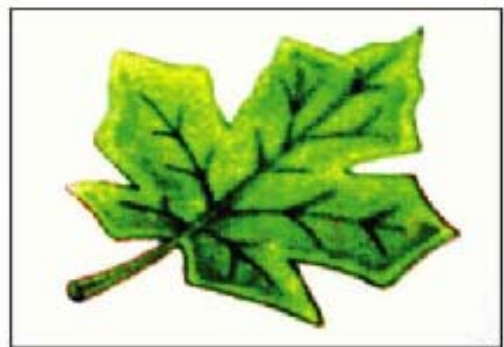

Leaf

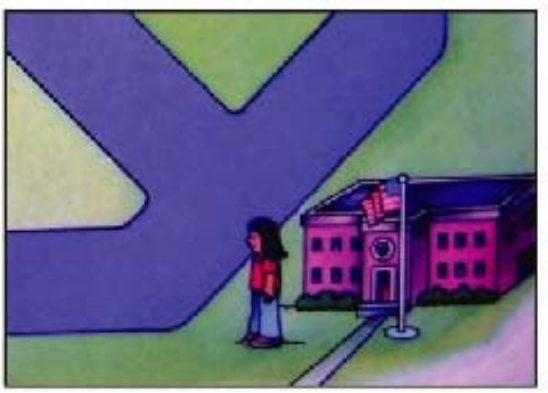

Part of original map (USA)

Fig. 1. Pictures from original BSID-II.

The words were translated into Bangla and some were even further modified based on the local dialects to keep the words that were familiar for both the urban and rural children. Tables 2 and 3 show the original and modified words. 
These responses obtained from Bangladeshi children show the difficulties due to culture biasness and unfamiliarity. The materials like picture book, rattle and sugar pellet were found unfamiliar for the children and therefore locally available similar materials were used to replace them.
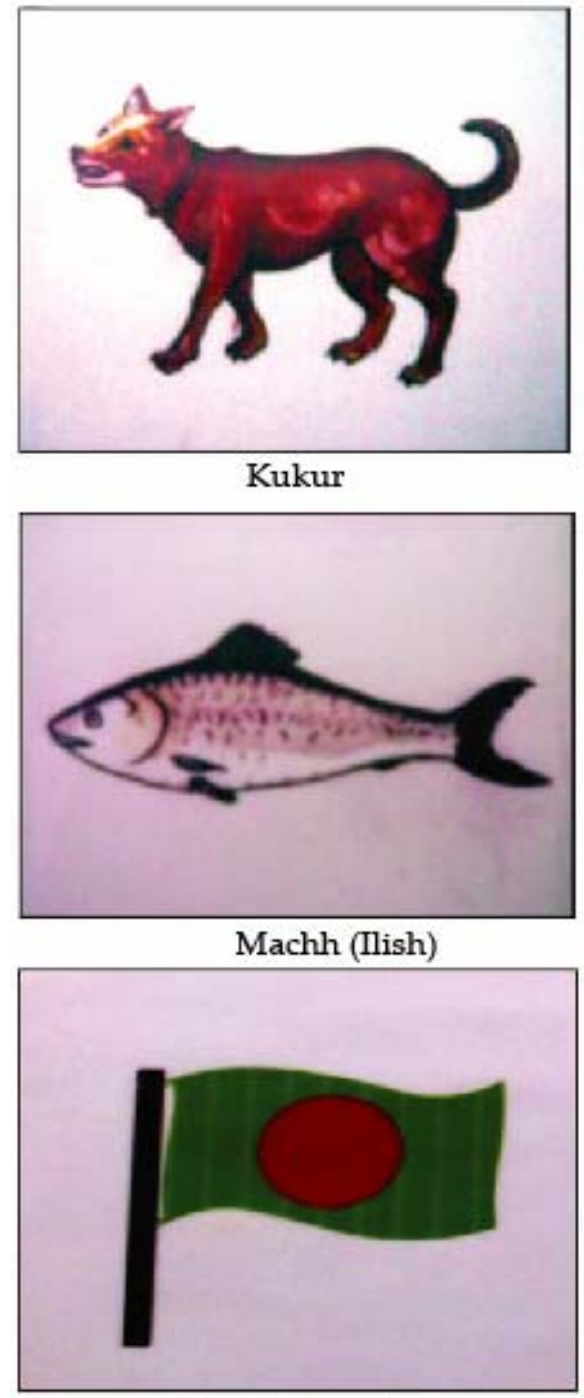

Potaka (Flag)

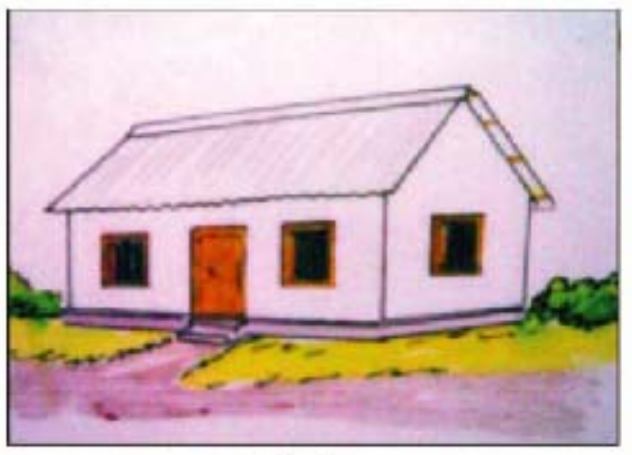

Bari

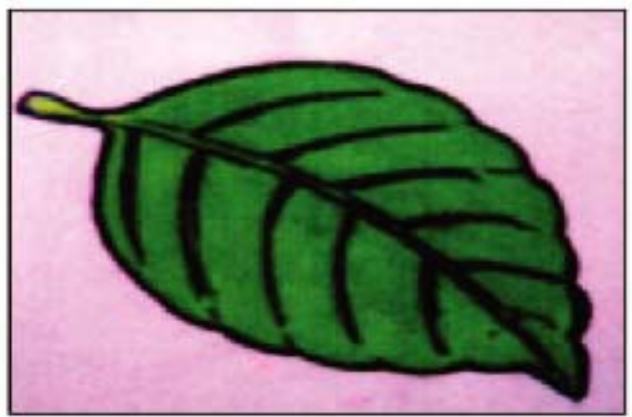

Pata

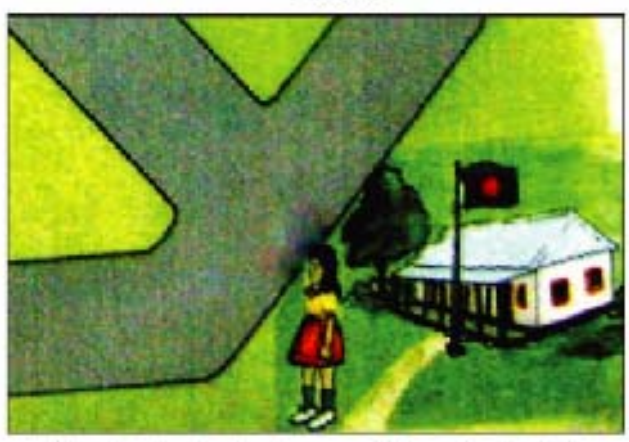

Part of adapted map (Bangladesh)

Fig. 2. Sample of picture adaptations.

In adaptation of BSID-II, the adaptation of the picture book required the maximum attention, particularly for the infants. The original book was from the USA named "Thomas, The Tank Engine Visits a Farm." However, the theme and pictures of this book 
were found to be unfamiliar for the infants and children of Bangladesh i.e. they were not be able to relate to the story. Based on experts' opinion local story with familiar pictures from the story 'Shishur Jotno' of the famous 'Meena Raju Series' was selected as appropriate for the Bangladeshi infants and children. By changing the story from the original to the local book, children were found to be more attentive and most children responded to the pictures spontaneously as they were familiar to them well ahead.

Table 2. Participants' responses to the original BSID- II test items.

\begin{tabular}{ll}
\hline Photo/ picture (original) & Different responses by Bangladeshi children (special responses) \\
\hline $\begin{array}{l}\text { Dog (puppy) } \\
\text { House with chimney }\end{array}$ & Cat, cow, fox, etc. \\
$\begin{array}{l}\text { Fish (pomfret) } \\
\text { Picture book }\end{array}$ & $\begin{array}{l}\text { Cartoon ( does not say anything), photo/picture } \\
\text { Less stimulating for the infants and children since the stories were } \\
\text { culture bound }\end{array}$ \\
\hline Performance items & Response by Bangladeshi children \\
\hline $\begin{array}{l}\text { Blue board } \\
\text { Pink board }\end{array}$ & $\begin{array}{l}\text { No interest; lack of experience/lack of lustre; do something else, } \\
\text { Other materials : }\end{array}$ \\
Baby doll (black baby) & Tends to avoid; looks away; \\
& some children were observed to be fearful of the black doll \\
\hline Discriminating items & Comments \\
\hline Apple & Banana more familiar \\
Calf (foreign) & Local small calf (বाছू,) \\
\hline
\end{tabular}

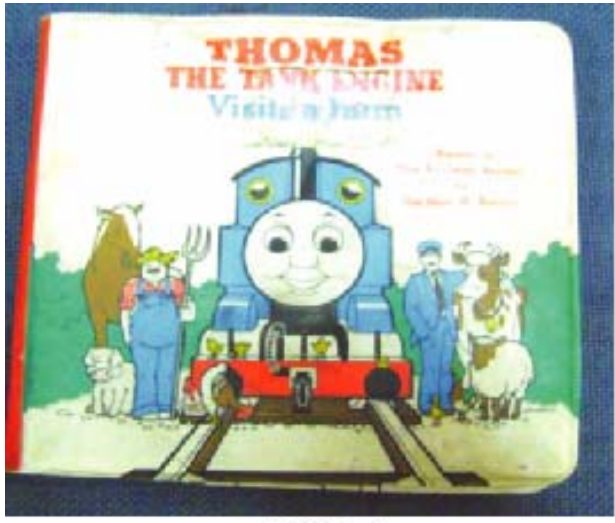

Original

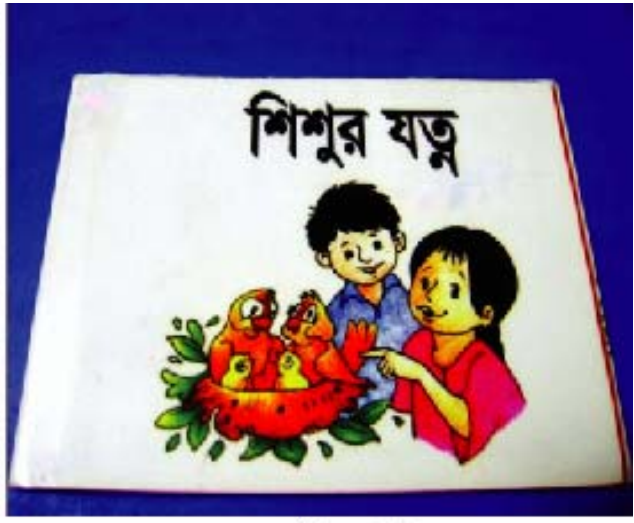

Adapted

Fig. 3. Cover page of the original and Bangla adapted picture books of BSID-II. 
Table 3. Material adaptations of BSID-II.

\begin{tabular}{ll}
\hline Material & Explanation \\
\hline Picture book & The original book is from USA “Thomas The Tank Engine Visits a Farm" \\
Small toy (rabbit) & $\begin{array}{l}\text { Instead of rabbit a small doll (boy or girl) of similar material and size was } \\
\text { used for some time. Some other small attractive toys can also be used }\end{array}$ \\
Rattle & $\begin{array}{l}\text { This is an easily breakable toy, so often needs to be replaced. Locally } \\
\text { produced similar size rattle with a handle was used. However, the sound }\end{array}$ \\
& $\begin{array}{l}\text { was usually loud. Very few rattle with soft sound was available } \\
\text { Sugar pellet }\end{array}$ \\
& were used
\end{tabular}

The Table 4 shows the word to word translation of a few words and adaptation by the experts in the context of Bangladesh.

Table 4. Word adaptation suggested by the expert panel.

\begin{tabular}{|c|c|}
\hline Shoe $=R$ \& v & Car = গাড়ি \\
\hline Sneaker $=m " v i ̂ \hat{j}$ & Auto $=$ ভো \\
\hline Tennis shoe $=R$ v ú my & Toy car = থেলনা গাড়ি \\
\hline Reebok $^{R}=e$ Wv & Vehicle/Toyota = টয়োটা \\
\hline Fish = মाছ & Pip pip = পিશ পિજ \\
\hline \multirow[t]{3}{*}{ Gold fish = ইলিশ মাছ } & Leaf $=$ পাতা/শাক \\
\hline & Fishie = হাগ্না \\
\hline & Blue gill \\
\hline
\end{tabular}

A renowned developmental pediatrician from the Institute of Child Health, UK visited Bangladesh and she carefully reviewed the research work and gave the following suggestions on the pictures drawn for the Bangladeshi infant and children.

1. The shape and theme of the original picture might be preserved while modifying for the children in Bangladeshi context.

2. The pictures might not be so bright i.e. the color and size of the modified pictures might be as near to the original as possible. The originals were developed after extensive testing by specialists in the field, so the changes only will reflect cultural variation but not the theme or subject or tone.

3. Bright pictures could be used for the children with "low vision".

4. For assessing children, soothing colors are more preferable.

Children gain a lot of knowledge from their surroundings. These knowledge and experiences vary from culture to culture, which can be observed from the child's responses. These variations in children's responses were not only seen in between country to country but can sometimes also be observed between urban and rural children of the same country, and this is due to differences in their social milieu. Moreover, 
environmental variations are also evident among urban and rural children and infants. The selection of the adapted words was done by the experts according to the responses of children during administration of the verbal items of BSID-II and rural urban dialectic differences were noted (such as a child could respond in his colloquial language). After seeing the picture of a leaf the immediate response from typical urban children was 'pata' (leaf), whereas the rural children called it 'shak' (spinach). Moreover, in the similar cases as that of a dog's picture, though the normal word for it is 'kukur' but the rural children call it 'kutta' (local language) as they were more familiar to this word. Numerous cases like these had also been encountered. These words were considered as alternatives to correct response for rural children. So, in this regard if a rural child responds to the object in the picture with its local name, it is also considered as a correct response (Table 5). Subsequently, further researches could be done on the environment influencing the child's language development.

Table 5. The responses from children in adapted picture sensitivity test.

\begin{tabular}{|c|c|c|}
\hline $\begin{array}{l}\text { Picture sensitivity } \\
\text { tests }\end{array}$ & $\begin{array}{l}\text { Children } \\
(\mathrm{N}=32)\end{array}$ & Responses \\
\hline $\begin{array}{l}\text { The final adapted } \\
\text { pictures were } \\
\text { displayed }\end{array}$ & $\begin{array}{l}3 \text { to } 4 \text { years old children studying in a } \\
\text { regular play-group and pre-primary } \\
\text { school. (This age limit i.e. from specific } \\
\text { age to a little bit older was taken as the } \\
\text { older ages reflect the developmental } \\
\text { matured response) }\end{array}$ & $\begin{array}{c}\text { They were attentive and } \\
\text { their responses were } \\
\text { positive }\end{array}$ \\
\hline
\end{tabular}

The first revised adaptation had the following corrections.

1. The pictures were too bright without following any set rules.

2. There were problems with proportion; e.g. the dogs had two small limbs/legs.

3. The quality of the drawings was also poor.

The above shortcomings were identified through group discussions and constructive criticisms ${ }^{(5)}$. These were corrected in subsequent version and administered in children who are in the respective age group. In the second revised adaptation the BSID-II pictures were made culture-free and familiar to the Bangladeshi infants and children.

When a foreign psychometric test is adapted for another country/culture, the adaptation lowers the cost of research, at the same time providing the measures that can be equally valid and reliable as the original instrument. It also allows carrying out comparisons at national and international levels using aggregate data. Moreover, it allows including the experience of the original authors and of other users ${ }^{(6)}$. The revision and adaptation of items of BSID-II for Bangladeshi infants will ensure all these lower cost, culturally sensitive, standardized instrument for assessing infant and child development. Further validation is required to use this scale in Bangladesh. 


\section{References}

1. Black MM and K Matula 2000. Essentials of Bayley Scales of Infant Development-II Assessment. John Wiley and Sons Inc, New York.

2. Walker SP, TD Wachs, S Grantham-McGregor, MM Black, CA Nelson, SL Huffman, H BakerHenningham, SM Chang, JD Hamadani, B Lozoff, JM Gardner, CA Powell, A Rahman and L Richter 2011. Inequality in early childhood: risk and protective factors for early child development. Lancet. 378: 1325-1338.

3. Anastasi A and S Urbina 1997. Psychological Testing. Singapore: Pearson Education.

4. Bayley N 1993. Manual for the Bayley Scales of Infant Development (2 ${ }^{\text {nd }}$ Ed.). San Antonio, TX: Psychological Corporation.

5. Malda M, FJR Van De Vijver, K Srinivasan, C Transler, P Sukumar, and K Rao 2008. Adapting a cognitive test for a different culture: An illustration of qualitative procedures. Psychology Science Quarterly. 50(4), 451-468.

6. Serra V Sutton and M Herdman 2001. Methodology of cross-cultural adaptation instruments for measuring health related quality of life. Catalan Agency for Health Information, Assessment and Quality. 